\title{
Heart Rate Variability in Patients with Hypertension: the Effect of Metabolic Syndrome and Antihypertensive Treatment
}

\author{
Małgorzata Maciorowska ${ }^{D}$, Paweł Krzesiński, Robert Wierzbowski, and Grzegorz Gielerak \\ Department of Cardiology and Internal Diseases, Military Institute of Medicine, Szaserow Street 128, 04-141 Warsaw 44, Poland \\ Correspondence should be addressed to Małgorzata Maciorowska; mmaciorowska@wim.mil.pl
}

Received 16 June 2020; Revised 10 September 2020; Accepted 25 September 2020; Published 14 October 2020

Academic Editor: Nicholas B. Norgard

Copyright (c) 2020 Małgorzata Maciorowska et al. This is an open access article distributed under the Creative Commons Attribution License, which permits unrestricted use, distribution, and reproduction in any medium, provided the original work is properly cited.

\begin{abstract}
Metabolic syndrome (MetS) is a combination of factors which, collectively, increase cardiovascular risk to a greater extent than each of them separately. Previous studies showed high cardiovascular risk to be associated with autonomic nervous system dysfunction. The purpose of this study was to assess the effects of antihypertensive treatment on heart rate variability (HRV) in patients with hypertension (HTN), depending on cooccurrence of MetS. 118 patients with uncontrolled HTN were enrolled to the study. HRV was compared among patients with and without MetS (MetS [+], $n=70$ ) at baseline and following 12 months antihypertensive treatment. The HRV indices measured from RR intervals recorded form using 24-hour ambulatory electrocardiography. The measured HRV domains were the standard deviation of the average of NN intervals [SDNN], square root of the mean of the sum of the squares of differences between adjacent NN intervals [rMSSD], percentage of NN50 [pNN50], low frequency [LF], high frequency [HF], total power of variance of all NN intervals [TP], and LF/HF ratio. Baseline parameters: SDNN, rMSSD, pNN50, and HF were significantly lower in the MetS[+] compared to the MetS[-] subgroup $(p<0.05)$. After a 12-month antihypertensive treatment, MetS[+] patients achieved a significant improvement in parameters: SDNN, rMSSD, pNN50, and TP $(p<0.05)$, while the changes in HRV observed in the MetS[-] subgroup were not statistically significant. The cooccurrence of HTN and other components of MetS is associated with disturbances of the autonomic balance. HTN control has a beneficial effect on HRV, with the effect being more evident in patients with MetS.
\end{abstract}

\section{Introduction}

Metabolic syndrome (MetS) is a combination of cardiovascular risk factors, such as abdominal obesity, hypertension (HTN), abnormal glucose metabolism, and atherogenic dyslipidemia (hypertriglyceridemia and low HDL levels) [1]. Combined, these factors were shown not only to adversely affect cardiovascular hemodynamics [2] but, above all, to increase cardiovascular risk to a greater extent than each of them individually [3-5].

MetS is very common in general population, but the prevalence is highly influenced by different diagnostic criteria used. According to the 2003-2012 data from NHANES prevalence of the MetS in the United States was 33\%, with prevalence growing with age, $18.3 \%$ among those $20-39$ years to $46.7 \%$ in those aged 60 years or older [6], the survey conducted in the Chinese elderly population showed increase prevalence of MetS from $50.4 \%$ in 2001 to $58.1 \%$ in 2010 [7]. European MetS prevalence, using the International Diabetes Federation diagnostic criteria, has been estimated as $41 \%$ in men and $38 \%$ in women [8]. Previous studies show that MetS is a significant predictor of cardiovascular morbidity and mortality $[3,4,8]$. Mediterranean Hypertensive Population patients with three or more components of MetS had threefold higher risk for cardiac events, 2.59 for cerebrovascular, and 2.26 for total cardiovascular events compared with those with no other component [9]. Consequently, the total cost of the complications of the syndrome including the cost of health care and loss of potential economic activity is huge.

Earlier studies demonstrated the complex pathophysiology of MetS by identifying the role of genetic and environmental factors, insulin resistance, inflammation, and oxidative stress $[10,11]$. Therefore, MetS is unequivocally a systemic condition. There are a number of papers showing 
MetS-associated autonomic nervous system (ANS) imbalance, manifesting as elevated sympathetic and diminished parasympathetic activity. This phenomenon has been observed for all MetS components, including HTN [12-14]. The sympathetic hyperexcitability appears to have primarily consequences for the development of obesity and insulin resistance as well as hypertension, what is connected with elevated urinary and plasma noradrenaline levels, TNF $\alpha$ contribution, elevation of adipokine levels, renal upregulation of glucose transporters, $\beta$-adrenoceptor sensitization, and angiotensin II release [15].

There are many methods that allow both direct and indirect assessment of the ANS function. An indirect method, relatively easily accessible, is evaluation of heart rate variability (HRV) in ambulatory electrocardiography. It has been postulated that a noninvasive assessment of ANS activity, e.g., via analyzing heart rate variability (HRV), may be useful in identifying patients at risk of developing MetS in the future [16]. Particularly, 24-hour recordings seem to be more reliable to clarify to what extent HRV is altered in MetS. HRV is frequently abnormal in patients with clinically overt cardiovascular conditions, such as coronary artery disease and heart failure $[17,18]$, strongly related to poorly controlled cardiometabolic risk factors.

There are studies which have examined the association between metabolic syndrome and heart variability, but fewer take the challenge to evaluate the effectiveness of applied treatment in primarily not treated hypertensives. Therefore, the purpose of this study was the assumption that in the case of patients with HTN and MetS, it seems of clinical importance to determine how much the concomitant metabolic disturbances affect HRV and whether or not antihypertensive treatment modifies HRV to the same extent as in hypertensive patients without MetS.

\section{Methods}

2.1. Study Population. This study analyzed the data collected from patients recruited for the FINEPATH research study (ClinicalTrials.gov Identifier NCT01996085), which had been conducted at the Department of Cardiology and Internal Diseases of Military Institute of Medicine, in the period 2011-2014. The FINEPATH study enrolled 144 patients with uncontrolled HTN, defined as elevated blood pressure ( $\geqq 140 / 90 \mathrm{mmHg}$ ) for at least 3 months prior to study enrollment, without pharmacotherapy at baseline. The key patient characteristics were presented in one of the earlier publications by our team [19]. The FINEPATH study was a prospective, randomized, controlled study to assess a novel HTN treatment. The following drug classes were used after baseline assessment: beta-blockers, angiotensin converting enzyme (ACE) inhibitors, angiotensin receptor blockers (ARB), calcium channel blockers (CCB), and diuretics, either alone or in combination. The final follow-up visit was conducted 12 months after treatment initiation. The exclusion criteria were (1) secondary HTN; (2) chronic kidney disease (glomerular filtration rate $(\mathrm{GFR})<60 \mathrm{~mL} / \mathrm{min} / 1.73 \mathrm{~m}^{2}$ calculated by the MDRD formula); (3) other severe comorbidities, including systolic heart failure, cardiomyopathy, significant arrhythmias, significant valvular heart disease, chronic obstructive pulmonary disease, previously diagnosed diabetes mellitus, polyneuropathy, and peripheral vascular disease; (4) age $<18$ years and $>75$ years; (5) body mass index $(\mathrm{BMI})>40 \mathrm{~kg} / \mathrm{m}^{2}$; (6) psychiatric disorders precluding the patient's cooperation; (7) any nonsinus hearth rhythm (including permanent cardiac pacing); and (8) ECG tracings containing $>300$ premature complexes and artifacts. The study protocol had been approved by the Institutional Review Board at Military Institute of Medicine (Approval No. 21/WIM/2011), and each patient had provided his or her written consent.

2.2. History and Physical Examination. History-taking and physical examination focused particularly on cardiovascular risk factors: age, sex, office systolic blood pressure (SBP), office diastolic blood pressure (DBP), smoking, family history of heart disease, and BMI. The following parameters were measured in each patient: fasting blood glucose $(\mathrm{mg} / \mathrm{dL})$, creatinine $(\mathrm{mg} / \mathrm{dL})$, high-density lipoprotein (HDL) cholesterol (mg/dL), low-density lipoprotein (LDL) cholesterol (mg/dL), triglyceride levels (mg/dL), and estimated GFR (MDRD eGFR) $\left(\mathrm{mL} / \mathrm{min} / 1.73 \mathrm{~m}^{2}\right)$. Metabolic syndrome was diagnosed based on the International Diabetes Federation (IDF) criteria [20]: central obesity-waist circumference $>94 \mathrm{~cm}$ for European men and $>80 \mathrm{~cm}$ for European women plus any two of the following four factors: triglyceride levels $\geq 150 \mathrm{mg} / \mathrm{dL}$ $(\geq 1.7 \mathrm{mmol} / \mathrm{L})$, or treatment for hypertriglyceridemia; HDL - cholesterol levels $<40 \mathrm{mg} / \mathrm{dL} \quad(<1.03 \mathrm{mmol} / \mathrm{L})$ in men or $<50 \mathrm{mg} / \mathrm{dL}(<1.29 \mathrm{mmol} / \mathrm{L})$ in women, or treatment for low HDL; SBP $\geq 130 \mathrm{mmHg}$ or $\mathrm{DBP} \geq 85 \mathrm{mmHg}$, or treatment for previously diagnosed HTN; fasting plasma glucose $\geq 100$ $\mathrm{mg} / \mathrm{dL} \quad(\geq 5.6 \mathrm{mmol} / \mathrm{l})$, or previously diagnosed diabetes mellitus.

2.3. 24-Hour Ambulatory Electrocardiography (Holter Monitoring). All patients underwent 24-hour ambulatory electrocardiography with 3-channel digital LifeCard CF recorders (Del Mar Reynolds Medical - Spacelabs Healthcare; US) to assess any arrhythmias, minimum, mean, maximum heart rate (HR), and HRV.

The time-domain and spectral (frequency-domain) HRV parameters were analyzed with the use of the Impresario Symphony Holter Analyzer system (Del Mar Reynolds Medical, Spacelabs Healthcare Ltd/UK). The preliminary processing of the obtained ECG tracings included a review and correction of wrongly classified beats, artifact elimination, and evaluation of any arrhythmias and ST-segment changes. Only the R-R intervals between normal QRS complexes were analyzed, with the R-R intervals preceding and following ventricular premature complexes excluded from analysis. The author who analyzed the HRV data was blinded to which patients had metabolic syndrome and which patients did not.

The patients were asked to avoid intense physical activity, smoking, and drinking alcohol. They were recommended to stop their activity at 10 p.m. and sleep till 6 a.m. The examinations were performed in hospital settings that limited the influences of other confounding factors, such as diet and work stress. 


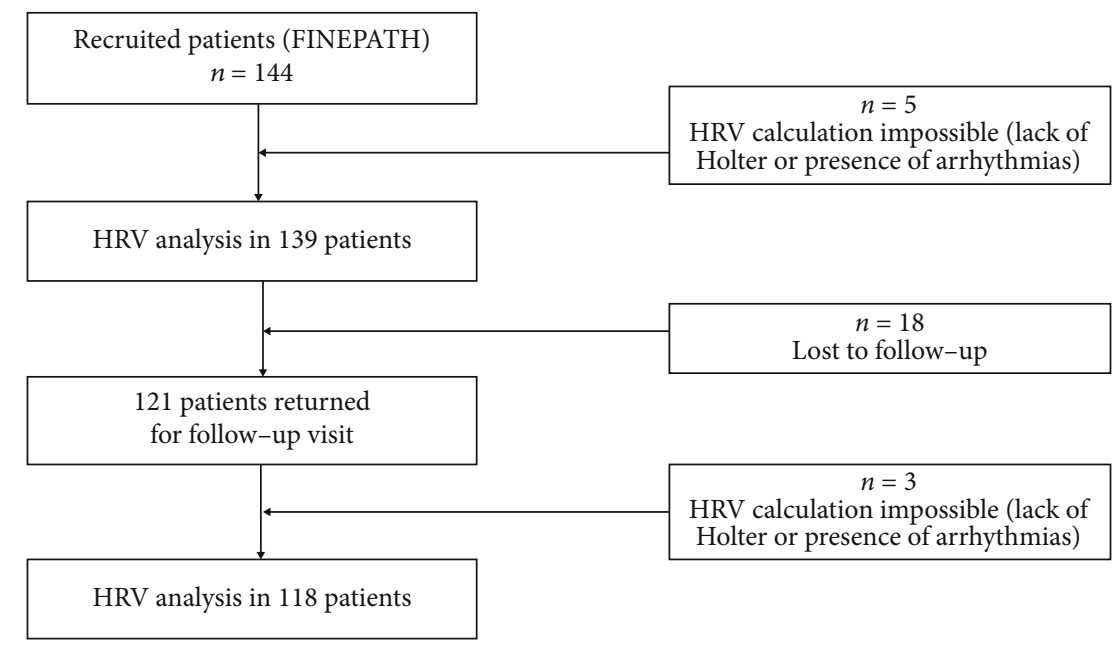

Figure 1: Study patient flow chart.

2.4. Analysis of HRV Time-Domain Parameters. The automatically detected time-domain parameters included in our analysis were daytime (parameter_day), nighttime (parameter_night), and 24-hour (parameter_24h) HRV parameters. The time-domain analysis of HRV provides mainly quantitative data, illustrating the extent of variability. The following parameters were used in our comprehensive HRV assessment: the standard deviation of the average of NN intervals in milliseconds (SDNN) and - for assessing the parasympathetic component in the area under the curve - the squares for assessing the parasympathetic component in the area under the curve-, the square root of the mean of the sum of the squares of differences between adjacent $\mathrm{NN}$ intervals in milliseconds (rMSSD), and the percentage of NN50 (pNN50) [21].

2.5. Analysis of Frequency-Domain HRV Parameters. The analysis of frequency-domain parameters was conducted with fast Fourier transform (FFT). Out of the total recorded spectrum, our analysis included the normalized low-frequency (LF) $(0.05-0.15 \mathrm{~Hz})$ and high-frequency $(\mathrm{HF})(0.15-0.4 \mathrm{~Hz})$ values, $\mathrm{LF} / \mathrm{HF}$ ratio, and total power of variance of all $\mathrm{NN}$ intervals [TP]). A spectral analysis was conducted for each hour out of the 24-hour period. Subsequently, the mean daytime and nighttime values were calculated and the day/night ratio. The HF parameter was considered to be an indicator of the parasympathetic activity. LF values depend on the effect of both the vagus nerve and sympathetic tone. At rest, LF shows a combined effect of the sympathetic and parasympathetic nervous systems, whereas following sympathetic stimulation (e.g., standing up, exercise, and psycho-emotional stress), LF reflects mainly the activity of the sympathetic nervous system. The relationship between $\mathrm{LF}$ and $\mathrm{HF}$ (LF/HF ratio) reflects the sympathetic-parasympathetic balance [21].

2.6. Statistical Analysis. Statistical analyses were conducted with Statistica 12.0 (StatSoft Inc.). The distribution and normality of data were assessed visually and with the KolmogorovSmirnov test. Continuous variables were presented as the mean \pm standard deviation (SD), whereas categorical variables were presented as absolute and relative values (percentages). A comparison analysis was conducted for two subgroups: MetS[+] (patients with other MetS factors apart from HTN) and MetS[-] (patients not diagnosed with MetS). Student's $t$-test was used for normally distributed data, whereas the Mann-Whiney U-test was used for the data with nonnormal distribution. Spearman's rank correlation coefficient was performed to investigate the relations between changes in $\mathrm{BP}$ and HRV parameters. The assessment of treatment effects for subgroups separately involved the use of the Wilcoxon signed-rank test. And the nonparametric Friedman test as an alternative to the two-way repeated measures ANOVA was performed in order to determine whether there is a significant interaction between MetS and effect of time (treatment). The $p$ value of $<0.05$ was considered statistically significant.

\section{Results}

Out of the 144 patients included in the FINEPATH study, 139 underwent Holter monitoring with HRV analysis, with data from 118 patients (who returned for the final followup visit) included in the final analysis. Eighteen patients had been lost to follow-up (they failed to return for the visit after 12 months), and the HRV of 3 patients could not be calculated due to a lack of Holter monitoring or the presence or arrhythmias) (Figure 1).

3.1. Baseline Characteristics. The study group comprised mostly males (69\%). The mean age was 46 years, mean HR $74 \mathrm{bpm}$, and mean blood pressure $141 / 90 \mathrm{mmHg}$. All patients enrolled to the study were Caucasian. More than half (59\%) of the patients met the MetS criteria (Table 1). Those subjects were slightly (borderline $p$ ) and more frequently males.

3.2. Comparison of Baseline HRV Values. Table 2 presents HRV parameters in patients stratified by the presence or absence of MetS prior to antihypertensive treatment initiation. In comparison with the MetS[-] subgroup, the patients from the MetS[+] subgroup of comparable age, HR, and blood pressure values showed significantly lower values of the following time-domain HRV parameters: SDNN_24h 
TABLE 1: Baseline patient characteristics (the entire study population).

\begin{tabular}{|c|c|c|c|c|}
\hline & All $[n=118]$ & MetS $[+], n=70$ & MetS [-], $n=40$ & $p$ \\
\hline Age [years] & $46 \pm 10$ & $48 \pm 10$ & $44 \pm 11$ & 0.054 \\
\hline Males & $80(68)$ & $55(79)$ & $25(52)$ & 0.002 \\
\hline $\mathrm{HR}[\mathrm{bpm}]$ & $74 \pm 11$ & $74 \pm 10$ & $73 \pm 12$ & 0.382 \\
\hline OSBP $[\mathrm{mmHg}]$ & $141 \pm 13$ & $142 \pm 11$ & $140 \pm 15$ & 0.284 \\
\hline ODBP $[\mathrm{mmHg}]$ & $90 \pm 9$ & $90 \pm 9$ & $89 \pm 10$ & 0.500 \\
\hline MetS (IDF) & $70(59)$ & $70(100)$ & $0(0)$ & - \\
\hline Creatinine $[\mathrm{mg} / \mathrm{dL}]$ & $0.83 \pm 0.16$ & $0.85 \pm 0.14$ & $0.82 \pm 0.19$ & 0.342 \\
\hline $\mathrm{eGFR}\left[\mathrm{mL} / \mathrm{min} / 1.73 \mathrm{~m}^{2}\right]$ & $99 \pm 17$ & $99 \pm 16$ & $99 \pm 19$ & 0.683 \\
\hline Glucose [mg/dL] & $99 \pm 12$ & $103 \pm 12$ & $93 \pm 7.2$ & $<0.001$ \\
\hline Total cholesterol $[\mathrm{mg} / \mathrm{dL}]$ & $225 \pm 39$ & $226 \pm 40$ & $222 \pm 37$ & 0.828 \\
\hline $\mathrm{HDL}[\mathrm{mg} / \mathrm{dL}]$ & $59 \pm 18$ & $52 \pm 15$ & $68 \pm 19$ & $<0.001$ \\
\hline $\mathrm{LDL}[\mathrm{mg} / \mathrm{dL}]$ & $144 \pm 34$ & $148 \pm 31$ & $139 \pm 39$ & 0.294 \\
\hline $\mathrm{TG}[\mathrm{mg} / \mathrm{dL}]$ & $152 \pm 76$ & $186 \pm 73$ & $103 \pm 48$ & $<0.001$ \\
\hline BMI $\left[\mathrm{kg} / \mathrm{m}^{2}\right]$ & $29 \pm 4$ & $30 \pm 4$ & $27 \pm 4$ & $<0.001$ \\
\hline Smokers & $23(19)$ & $10(21)$ & $13(19)$ & 0.761 \\
\hline Statin & $3(2.5)$ & $3(4)$ & $0(0)$ & 0.146 \\
\hline
\end{tabular}

Data presented as mean \pm SD/n (\%). BM: body mass index; eGFR: estimated glomerular filtration rate; HR: heart rate; IDF: International Diabetes Foundation criteria; MetS: metabolic syndrome; ODBP: office diastolic blood pressure; OSBP: office systolic blood pressure; TG: triglycerides.

$(p=0.048)$, SDNN_day $(p=0.015)$, rMSSD_24h $(p=0.002)$, rMSSD_day $(p=0.002)$, rMSSD_night $(p=0.020)$, pNN50_ $24 \mathrm{~h}(p=0.0008)$, pNN50_day $(p=0.0004)$, pNN50_night $(p=0.018)$, and the spectral parameter HF_night $(p=0.041)$.

3.3. Assessment of 12-Month Treatment Effects. After 12months of antihypertensive treatment, there was significant reduction in blood pressure both in the MetS[+] and MetS[-] subgroups, down to $120.1 / 77.2 \mathrm{mmHg}(p<0.001)$ and $121.2 / 77.6 \mathrm{mmHg}(p<0.001)$, respectively. A similar effect was also observed in terms of $\mathrm{HR}$, with $\mathrm{HR}$ of $69.1 \mathrm{bpm}(p<0.001)$ and $66.5 \mathrm{bpm}(p<0.001)$, respectively. The BP control $(<140 / 90 \mathrm{mmHg})$ was achieved in $68(97 \%)$ pts with MetS and $42(88 \%)$ pts without MetS $(p=0.041)$. The 12-month follow-up showed no changes in body weight either in the MetS[+] $(91.1 \pm 13.5 \mathrm{~kg}$ before vs. $91.3 \pm 13.4 \mathrm{~kg}$ after treatment; $p=0.73)$ or in the MetS[-] subgroup $(87.0 \pm 15.9 \mathrm{~kg}$ before vs. $86.6 \pm 15.3 \mathrm{~kg}$ after treatment; $p=0.84)$.

Antihypertensive monotherapy was used in $47.1 \%$ of patients (a CCB in $0.8 \%$, beta-blocker in $5.0 \%$, $\mathrm{ARB}$ in $4.2 \%$, diuretic in $3.4 \%$, ACE inhibitor in $33.6 \%$ ). Combination antihypertensive therapy was used in $50.4 \%$ of patients, with two-drug combination regimens of ACE inhibitor plus diuretic in $16.8 \%$ of patients, ACE inhibitor plus beta blocker in $10.9 \%$, ACE inhibitor plus CCB in $7.6 \%$, ARB plus diuretic in $3.4 \%$, ARB plus beta-blocker in $0.8 \%$, and ARB plus CCB in $0.8 \%$. Three-drug combination regimens, used in $7.5 \%$ of patients, included ACE inhibitor plus beta-blocker plus CCB in $2.5 \%$ of patients, $\mathrm{ARB}$ plus $\mathrm{CCB}$ plus diuretic in $0.8 \%$, ACE inhibitor plus beta-blocker plus diuretic in $2.5 \%$, and $\mathrm{CCB}$ plus betablocker plus diuretic in $1.7 \%$. No antihypertensive medica-
TABLE 2: Comparison of HRV parameters in subgroups stratified by concomitant MetS (before treatment initiation).

\begin{tabular}{lccc}
\hline & $\begin{array}{c}\text { MetS [+], } n=70 \\
\text { Mean } \pm \text { SD }\end{array}$ & $\begin{array}{c}\text { MetS [-], } n=48 \\
\text { Mean } \pm \text { SD }\end{array}$ & $p$ value \\
\hline SDNN_24h [ms] & 135.5 & 149.1 & 0.048 \\
SDNN_day [ms] & 109.8 & 121.5 & 0.015 \\
SDNN_night [ms] & 90.5 & 94.6 & 0.473 \\
rMSSD_24h [ms] & 31.5 & 38.2 & 0.002 \\
rMSSD_day [ms] & 27.3 & 32.7 & 0.002 \\
rMSSD_night [ms] & 40.0 & 49.0 & 0.020 \\
pNN50_24h [\%] & 6.80 & 10.79 & 0.0008 \\
pNN50_day [\%] & 4.66 & 7.63 & 0.0004 \\
pNN50_night [\%] & 12.65 & 19.28 & 0.018 \\
LF/HF_day [-] & 4.50 & 3.88 & 0.625 \\
LF/HF_night [-] & 3.04 & 2.01 & 0.048 \\
LF_day [n.u.] & 70.5 & 70.0 & 0.939 \\
LF_night [n.u.] & 62.3 & 55.8 & 0.061 \\
HF_day [n.u.] & 23.0 & 24.1 & 0.524 \\
HF_night [n.u.] & 32.3 & 38.3 & 0.041 \\
TP_day [ms ${ }^{2}$ ] & 2.747 & 3.248 & 0.152 \\
TP_night [ms ${ }^{2}$ ] & 2.923 & 3.174 & 0.616 \\
\hline
\end{tabular}

DBP: diastolic blood pressure; HF: power in the high frequency range; HR: heart rate; LF: power in the low frequency range; n.u. : normalized units; pNN50: percentage of NN50; rMSSD: square root of the mean of the sum of the squares of differences between adjacent NN intervals; SBP: systolic blood pressure; SDNN: standard deviation of the average of NN intervals; TP: total power of variance of all NN intervals.

tions were used in $2.3 \%$ of patients, who only received nonpharmacological recommendations. Statins were introduced in 16 patients with MetS (22.9\%). 
TABLE 3: Comparison of HRV parameters before and after 12-month treatment, in patients with HTN stratified by concomitant MetS.

\begin{tabular}{|c|c|c|c|c|c|c|c|c|}
\hline & $\begin{array}{c}\text { MetS[+], } n=70 \\
\text { Mean } \pm \text { SD } \\
\text { Before }\end{array}$ & $\begin{array}{c}\text { MetS[+], } n=70 \\
\text { Mean } \pm \text { SD } \\
\text { After }\end{array}$ & $\begin{array}{l}\text { Difference } \\
(\text { MetS[+]) }\end{array}$ & $p$ value & $\begin{array}{c}\text { MetS[-], } n=48 \\
\text { Mean } \pm \text { SD } \\
\text { Before }\end{array}$ & $\begin{array}{c}\text { MetS[-], } n=48 \\
\text { Mean } \pm \text { SD } \\
\text { After }\end{array}$ & $\begin{array}{l}\text { Difference } \\
\text { (MetS[-]) }\end{array}$ & $p$ value \\
\hline $\mathrm{HR}$ [L/min] & 74.1 & 69.1 & -5.0 & 0.023 & 72.3 & 66.5 & -5.8 & 0.007 \\
\hline OSBP $[\mathrm{mmHg}]$ & 142.1 & 120.1 & -22.0 & $<0.0001$ & 139.5 & 121.2 & -18.3 & $<0.0001$ \\
\hline ODBP [mmHg] & 90.5 & 77.2 & -13.3 & $<0.0001$ & 89.3 & 77.6 & -11.7 & $<0.0001$ \\
\hline SDNN_24h [ms] & 135.5 & 140.2 & 4.7 & 0.012 & 149.1 & 146.3 & -2.8 & 0.665 \\
\hline SDNN_day [ms] & 109.8 & 114.6 & 5.1 & 0.042 & 121.5 & 123.2 & 1.7 & 0.312 \\
\hline SDNN_night [ms] & 90.5 & 95.2 & 4.7 & 0.189 & 94.6 & 96.7 & 2.1 & 0.885 \\
\hline rMSSD_24h [ms] & 31.5 & 34.7 & 3.2 & 0.003 & 38.2 & 39.4 & 1.2 & 0.470 \\
\hline rMSSD_day [ms] & 27.3 & 29.7 & 2.4 & 0.001 & 32.7 & 35.0 & 2.3 & 0.855 \\
\hline rMSSD_night [ms] & 40.0 & 43.8 & 3.8 & 0.042 & 49.0 & 48.6 & -0.4 & 0.112 \\
\hline pNN50_24h [\%] & 6.80 & 8.03 & 1.23 & 0.0002 & 10.79 & 11.42 & 0.69 & 0.665 \\
\hline pNN50_day [\%] & 4.66 & 5.56 & 0.90 & 0.001 & 7.63 & 8.91 & 1.28 & 0.885 \\
\hline pNN50_night [\%] & 12.65 & 14.29 & 1.64 & 0.051 & 19.28 & 18.13 & -1.15 & 0.105 \\
\hline LF/HF_day [-] & 4.50 & 4.02 & -0.48 & 0.082 & 3.88 & 3.64 & -0.24 & 0.136 \\
\hline LF/HF_night [-] & 3.04 & 2.41 & -0.63 & 0.550 & 2.01 & 1.88 & -0.13 & 0.470 \\
\hline LF_day [n.u.] & 70.5 & 67.9 & -2.6 & 0.457 & 70.0 & 66.1 & -3.9 & 0.136 \\
\hline LF_night [n.u.] & 62.3 & 59.9 & -2.4 & 0.403 & 55.8 & 53.7 & -2.1 & 0.470 \\
\hline HF_day [n.u.] & 23.0 & 26.1 & 3.1 & 0.082 & 24.1 & 28.4 & 4.3 & 0.074 \\
\hline HF_night [n.u.] & 32.3 & 34.6 & 2.3 & 0.189 & 38.3 & 40.8 & 2.5 & 0.470 \\
\hline TP_day $\left[\mathrm{ms}^{2}\right]$ & 2.747 & 3.803 & 1.056 & 0.026 & 3.248 & 4.054 & 806 & 0.307 \\
\hline TP_night $\left[\mathrm{ms}^{2}\right]$ & 2.923 & 3.483 & 560 & 0.402 & 3.174 & 3.377 & 203 & 0.665 \\
\hline
\end{tabular}

ODBP: office diastolic blood pressure; HF: power in the high frequency range; HR: heart rate; LF: power in the low frequency range; n.u.: normalized units; pNN50: percentage of NN50; rMSSD: square root of the mean of the sum of the squares of differences between adjacent NN intervals; OSBP: office systolic blood pressure; SDNN: standard deviation of the average of NN intervals; TP: total power of variance of all NN intervals.

Table 3 presents a comparison of HRV parameters in patients with HTN stratified by the presence or absence of concomitant MetS at 12 months of antihypertensive treatment. MetS[+] patients achieved a significant improvement in their $\mathrm{HRV}$ as shown by time-domain parameters: SDNN_24h $(p=0.012)$, SDNN_day $(p=0.042)$, rMSSD_24h $(p=0.003)$, rMSSD_day $(p=0.001)$, rMSSD_ night $(p=0.042)$, pNN50_24h $(p=0.0002)$, pNN50_day $(p=0.001)$, and the frequency-domain parameter of TP_day $(p=0.026)$. The results achieved in the MetS[-] subgroup also suggest a favorable effect of treatment; however, the observed differences did not reach the adopted level of significance and were lower than in the MetS[+] subgroup also in terms of absolute values (Table 3 and Figure 2). Friedman's test revealed the significant interaction between MetS and effect of treatment for SDNN_day, rMSSD_day, pNN50_24h, pNN50_day, and TP_day. The significant correlations were observed for 12-month changes in diastolic blood pressure and some HRV parameters (SDNN, rMSDD, and pNN50) in both MetS[+] and MetS[-]. The effect on systolic blood pressure was less related to HRV [Supplementary Table 1].

\section{Discussion}

Our findings indicate a considerable effect of metabolic disorders on the HRV in patients with HTN. Implemented hypertensive therapy was effective in both subgroups, but the MetS patients were those who seem to benefit more from the treatment with respect to sympatovagal balance.

The baseline values of HRV parameters obtained in our study and the impact of MetS are consistent with the data reported in the available literature [12-14, 22-28]. A 2013 study by $\mathrm{Li}$ et al., which aimed to assess the relationship between MetS severity and ANS function, demonstrated independent negative correlations of two MetS components (fasting plasma glucose and HTN) with ANS function [23]. Moreover, an earlier study (Twins Heart Study) conducted in 288 pairs of twins showed a relationship between MetS and decreased HRV parameters, both in individual analyses and in the analyses of the twin pairs. Additionally, HRV parameters were found to be decreased in individuals with more MetS components [24]. American researchers [25] reached a similar conclusion while assessing the HR and HRV parameters in patients stratified by their fasting glucose (FG) levels and other concomitant MetS components. This American study demonstrated lowering of most of the evaluated HRV parameters (particularly the SDNN, standard deviation of the 5-minute average NN intervals [SDANN], TP, ultra-low frequency [ULF], and very low frequency [VLF] power) in patients with markedly elevated FG (6.1$6.9 \mathrm{mmol} / \mathrm{L}$ ) and type 2 diabetes (with FG $>6.9 \mathrm{mmol} / \mathrm{L}$ or on antidiabetic medication or insulin) in comparison with the patients with normal $(4.5-5.5 \mathrm{mmol} / \mathrm{L})$ and slightly elevated $(5.6-6.0 \mathrm{mmol} / \mathrm{L}) \mathrm{FG}$. The patients with normal to 


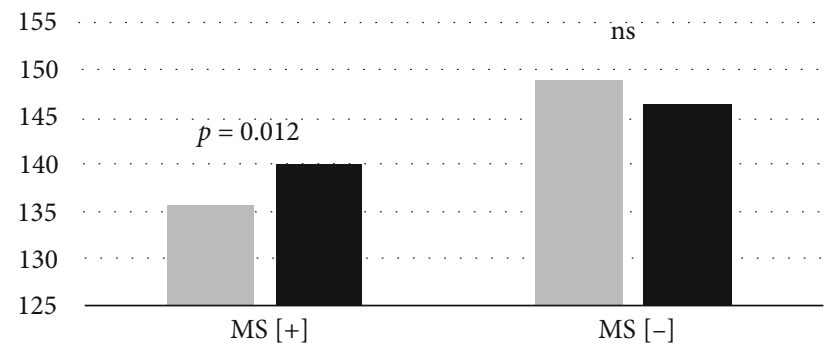

- SDNN_24h [ms] before treatment

- SDNN_24 h [ms] after treatment

45

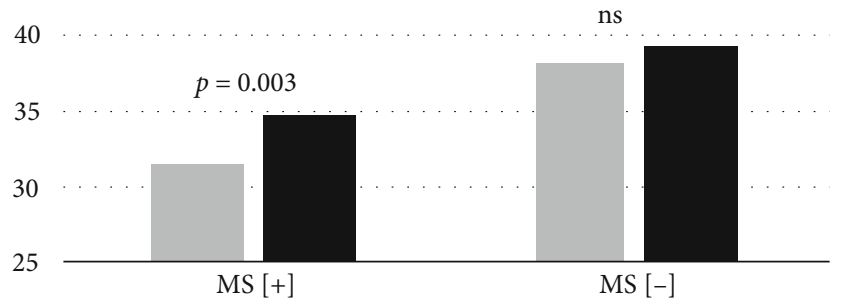

- rMSSD_24 h [ms] before treatment

- rMSSD_24 h [ms] after treatment 12

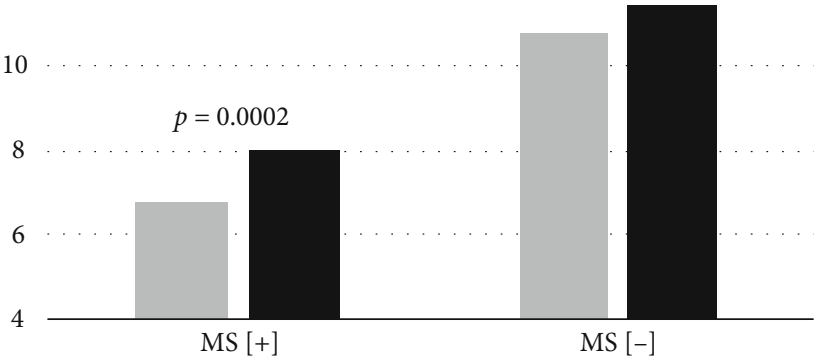

- pNN50_24 h [ms] before treatment

- pNN50_24 h [ms] after treatment

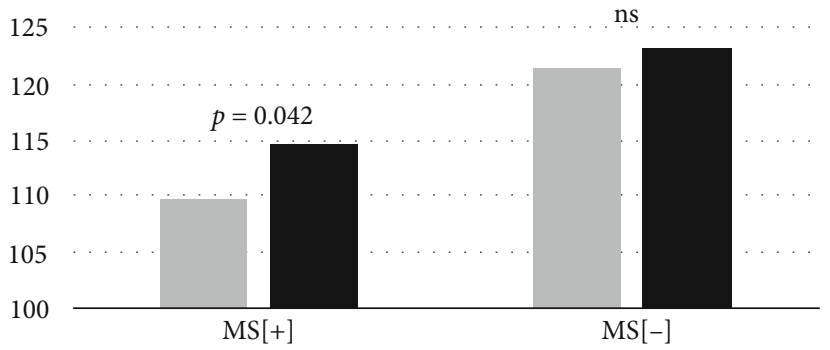

- SDNN_day [ms] before treatment

- SDNN_day [ms] after treatment

40

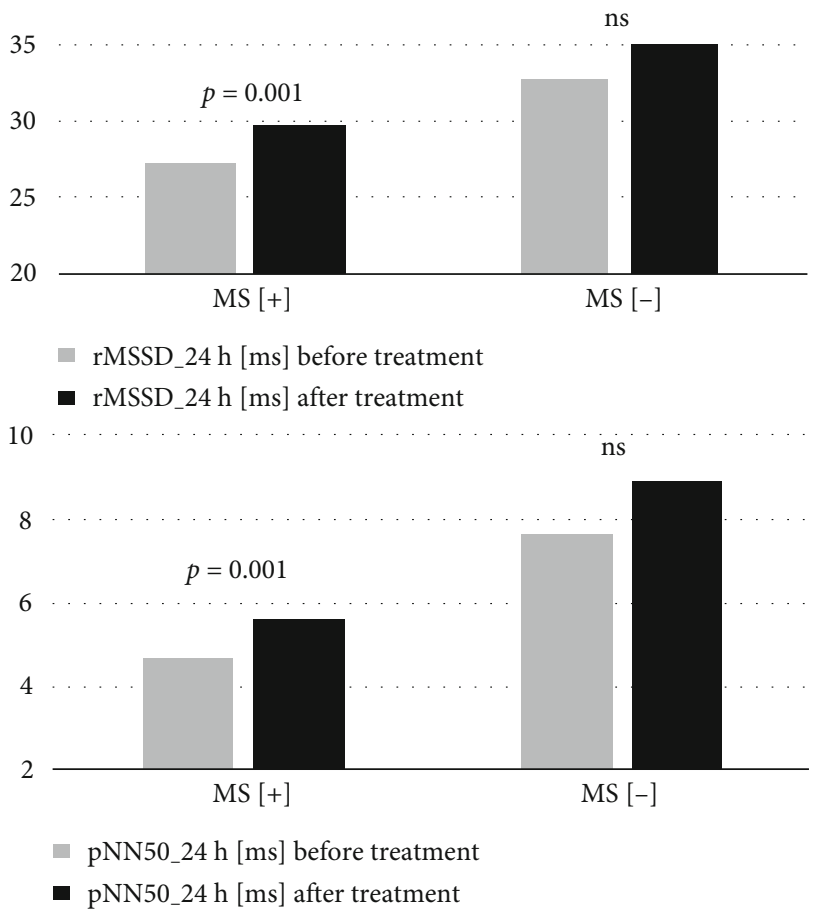

FIGURE 2: Comparison of selected HRV parameters before and after 12-month treatment, in patients with HTN stratified by concomitant MetS.

slightly elevated FG who met more than 2 MetS criteria showed decreased HRV (SDNN, SDANN, TP, and ULF) in comparison with the patients meeting at most one MetS criterion. In patients with diabetes or markedly elevated FG, MetS was associated with decreased HRV compared with the HRV in patients without MetS. These American findings were consistent with those of the Finnish authors whose 1998 study demonstrated significantly decreased HRV parameters (SDANN, TP, VLF, LF) in hypertensive patients with insulin resistance in comparison with both hypertensive patients without insulin resistance and normotensive patients. The HF parameter $(p<0.001)$ and baroreflex sensitivity $(p<0.05)$ were diminished in both hypertensive groups [28]. A prospective study by Balcioğlu et al. [29] $(n=240)$ showed significantly decreased HRV parameters (SDNN, SDNN index, SDANN, rMSSD, pNN50) in 24-hour Holter recordings in comparison with those in the control group. Unlike in the studies mentioned above, the lowering of
HRV parameters correlated only with fasting glucose levels, with no differences between the groups in terms of the remaining 4 diagnostic criteria of MetS (notably, both study groups included patients with HTN).

Our study demonstrated the effects of antihypertensive treatment on HRV parameters to be beneficial, particularly in the group of patients with MetS. The more altered HRV at baseline may partly explain greater reduction after 12 months of treatment in MetS. No other mechanism can be identified basing on our data. Our findings are consistent with earlier reports indicating beneficial effects of antihypertensive treatment on HRV. However, there is no clear consensus which hypotensive drugs are the most beneficial in terms of the sympatovagal balance. Some earlier studies demonstrated beta-blockers and ARB to be particularly beneficial in that respect [30-32]. Moreover, some other reports indicated that ARB treatment yielded better effects than treatment with ACE inhibitors and beta-blockers [33-35]. One 
prospective, randomized Japanese study compared the effects of ARB treatment in MetS patients randomized into three therapeutic groups (telmisartan, candesartan, diet therapy) [35]. At 6 months, the study showed a comparable lowering of blood pressure in both drug-treated groups. However, ARB treatment yielded increased baroreflex sensitivity, increased high-molecular-weight adiponectin levels, and improved endothelial dysfunction (in this last respect, a more pronounced effect was achieved with telmisartan). Moreover, the telmisartan group showed significantly decreased norepinephrine levels, blood pressure variability, and the spectral HRV parameter of the LF/HF ratio $(p<0.05)$. A study by Menzes et al. showed improvement in all HRV parameters (SDNN, pNN50, LF; $p<0.001$ ) following a 3-month treatment with an ACE inhibitor (enalapril or ramipril) in contrast with the control group [31]. Petretta et al. assessed the effect of a 12-month lisinopril treatment on HRV and, for the entire study population, observed an increase only in the nighttime HF parameter in comparison with baseline values [33]. However, the subgroup of patients with left ventricular mass normalization showed increased both daytime and nighttime HF, as well as increased nighttime TP and VLF. A 2010 study by Pavithran et al. examined 150 patients newly diagnosed with HTN, divided into five 30 patient groups, each receiving one of the following: amlodipine, atenolol, enalapril, hydrochlorothiazide, or an amlodipine+atenolol combination. Only the amlodipine+atenolol group showed a significant change in HRV (increased total variability of RR intervals and HF spectral power) [36]. There were also studies attempting to evaluate the effect of individual CCB medicines on HRV parameters [37-40]. The available data on the effect of amlodipine on the HRV are contradictory. Individual authors report either an insignificant-to-absent effect of this drug on the ANS activity, or enhanced sympathetic activity, or-conversely-vagus nerve stimulation [37-39]. A prospective, randomized study by Karas et al. $(n=57)$ evaluated the effects of treatment with amlodypine, ramipril, and telmistartan on HRV spectral analysis and plasma norepinephrine and epinephrine level measurements [41]. Following amlodipine treatment, an increased daytime sympathetic activity and decreased nighttime parasympathetic activity, together with increased plasma norepinephrine levels, were observed. Telmisartan treatment yielded considerably increased parasympathetic activity without changes in plasma norepinephrine levels, whereas ramipril increased the parasympathetic activity only during the day.

For both baseline comparison and treatment effects, time-domain HRV parameters revealed to better diversify the presence of metabolic burden than frequency-domain HRV parameters. The frequency-domain HF power is assumed to correspond to the frequency of breathing, reflecting respiratory sinus arrhythmia. More controversies concern LF power. Some authors undermine that LF power is and index of cardiac sympathetic tone and are even more willing to claim that it reflects baroreflexes [42].

Considering high prevalence of MetS around the world, our finding may concern a wide range of patients. Blood pressure control seems to complement the intervention based on diet and physical activity to reverse MetS and prevent cardiac autonomic neuropathy [43].

4.1. Strengths and Limitations. The strength of our study is the enrollment of hypertensive subjects, some of them with MetS, but no significant comorbidities. Moreover, there were no bias of previous hypotensive treatment at baseline assessment. Some limitations should be also considered. One is the small size of the study population and thus a small size of individual subgroups. Therefore, our analyses may be underpowered, and the findings need to be confirmed in a larger study group. Another limitation is the difference in sex proportions between MetS subgroups that could bias baseline comparison. Moreover, we would like to point out that the absence of any monitoring in terms of pharmacotherapy, other than the patients' antihypertensive treatment report, may have affected our results. Our study assessed neither patients' physical activity nor the effect of treatment on the metabolic dysfunction. In terms of Discussion, we would like to emphasize the issues with comparing individual studies due to the differences in study protocols, the adopted diagnostic criteria of MetS, and the length of analyzed electrocardiographic recordings. Moreover, only a handful of studies included separate groups of patients with uncomplicated HTN and those with HTN and concomitant metabolic disorders.

\section{Conclusions}

Our findings confirm that the cooccurrence of HTN and other components of MetS is associated with differences in HRV and its modulation by hypotensive medicines. Blood pressure control has a beneficial effect on HRV, with the effect being more evident in patients with MetS.

\section{Data Availability}

The datasets used and analysed during the current study are available from the corresponding author on reasonable request.

\section{Conflicts of Interest}

The authors declare that there is no conflict of interest regarding the publication of this paper.

\section{Acknowledgments}

The authors would like to thank the team from the Department of Cardiology and Internal Diseases who took part in recruiting patients and collecting data for analysis. The FINEPATH project was financed from the Military Institute of Medicine/Ministry of Science and Higher Education research grant (No. 148/WIM). 


\section{Supplementary Materials}

Supplementary Table 1. The correlations between changes (delta) in BP and HRV parameters after 12-month treatment. (Supplementary Materials)

\section{References}

[1] K. G. M. M. Alberti, P. Zimmet, and J. Shaw, "Metabolic syndrome-a new world-wide definition. A consensus statement from the international diabetes federation," Diabetic Medicine, vol. 23 , no. 5, pp. 469-480, 2006.

[2] P. Krzesiński, A. Stańczyk, K. Piotrowicz, G. Gielerak, B. Uziębło-Zyczkowska, and A. Skrobowski, "Abdominal obesity and hypertension: a double burden to the heart," Hypertension Research, vol. 39, no. 5, pp. 349-355, 2016.

[3] A. Scuteri, S. S. Najjar, C. H. Morrell, and E. G. Lakatta, "The metabolic syndrome in older individuals: prevalence and prediction of cardiovascular events: the cardiovascular health study," Diabetes Care, vol. 28, no. 4, pp. 882-887, 2005.

[4] P. W. F. Wilson and J. B. Meigs, "Cardiometabolic risk: a Framingham perspective," International Journal of Obesity, vol. 32, no. S2, pp. S17-S20, 2008.

[5] J. D. Tune, A. G. Goodwill, D. J. Sassoon, and K. J. Mather, "Cardiovascular consequences of metabolic syndrome," Translational Research, vol. 183, pp. 57-70, 2017.

[6] M. Aguilar, T. Bhuket, S. Torres, B. Liu, and R. J. Wong, "Prevalence of the metabolic syndrome in the United States, 2003-2012," JAMA, vol. 313, no. 19, pp. 1973-1974, 2015.

[7] M. Liu, J. Wang, B. Jiang et al., "Increasing prevalence of metabolic syndrome in a Chinese elderly population: 2001-2010," PLoS One, vol. 8, no. 6, article e66233, 2013.

[8] The DECODE Study Group, "Does the constellation of risk factors with and without abdominal adiposity associate with different cardiovascular mortality risk?," International Journal of Obesity, vol. 32, no. 5, pp. 757-762, 2008.

[9] E. Andreadis, G. Tsourous, C. Tzavara et al., "Metabolic syndrome and incident cardiovascular morbidity and mortality in a Mediterranean hypertensive population," American Journal of Hypertension, vol. 20, no. 5, pp. 558-564, 2007.

[10] S. L. Samson and A. J. Garber, "Metabolic syndrome," Endocrinology and Metabolism Clinics of North America, vol. 43, no. 1, pp. 1-23, 2014.

[11] M. Tanaka, "Improving obesity and blood pressure," Hypertension Research, vol. 43, no. 2, pp. 79-89, 2020.

[12] M. I. Stuckey, M. P. Tulppo, A. M. Kiviniemi, and R. J. Petrella, "Heart rate variability and the metabolic syndrome: a systematic review of the literature," Diabetes/Metabolism Research and Reviews, vol. 30, no. 8, pp. 784-793, 2014.

[13] L. R. Wulsin, P. S. Horn, J. L. Perry, J. M. Massaro, and R. B. D'Agostino, "Autonomic imbalance as a predictor of metabolic risks, cardiovascular disease, diabetes, and mortality," The Journal of Clinical Endocrinology and Metabolism, vol. 100, no. 6, pp. 2443-2448, 2015.

[14] J. F. Thayer, S. S. Yamamoto, and J. F. Brosschot, "The relationship of autonomic imbalance, heart rate variability and cardiovascular disease risk factors," International Journal of Cardiology, vol. 141, no. 2, pp. 122-131, 2010.

[15] E. Lemche, O. S. Chaban, and A. V. Lemche, "Neuroendorine and epigentic mechanisms subserving autonomic imbalance and HPA dysfunction in the metabolic syndrome," Frontiers in Neuroscience, vol. 10, p. 142, 2016.

[16] L. R. Wulsin, P. S. Horn, J. L. Perry, J. M. Massaro, and R. B. D'Agostino Sr., "The contribution of autonomic imbalance to the development of metabolic syndrome," Psychosomatic Medicine, vol. 78, no. 4, pp. 474-480, 2016.

[17] K. C. Bilchick, B. Fetics, R. Djoukeng et al., "Prognostic value of heart rate variability in chronic congestive heart failure (veterans affairs' survival trial of antiarrhythmic therapy in congestive heart failure)," The American Journal of Cardiology, vol. 90, no. 1, pp. 24-28, 2002.

[18] R. E. Kleiger, J. P. Miller, J. T. Bigger, and A. J. Moss, "Decreased heart rate variability and its association with increased mortality after acute myocardial infarction," The American Journal of Cardiology, vol. 59, no. 4, pp. 256-262, 1987.

[19] P. Krzesiński, G. Gielerak, A. Stańczyk et al., "The effect of hemodynamically-guided hypotensive therapy in one-year observation: Randomized, prospective and controlled trial (FINEPATH study)," Cardiology Journal, vol. 23, no. 2, pp. 132-140, 2016.

[20] IDF Clinical Guidelines Task Force, "Global guideline for type 2 diabetes: recommendations for standard, comprehensive, and minimal care," Diabetic Medicine, vol. 23, no. 6, pp. 579-593, 2006.

[21] M. Malik, J. T. Bigger, G. Breithardt et al., "Task force of the European Society of Cardiology and the North American Society of Pacing and Electrophysiology Heart Rate Variability. Standards of Measurement, Physiological Interpretation, and Clinical Use," European Heart Journal, vol. 17, pp. 354-381, 1996.

[22] D. Liao, R. P. Sloan, W. E. Cascio et al., "Multiple metabolic syndrome is associated with lower heart rate variability. The atherosclerosis risk in communities study," Diabetes Care, vol. 21, no. 12, pp. 2116-2122, 1998.

[23] Z. Li, Z. H. Tang, F. Zeng, and L. Zhou, “Associations between the severity of metabolic syndrome and cardiovascular autonomic function in a Chinese population," Journal of Endocrinological Investigation, vol. 36, no. 11, pp. 993-999, 2013.

[24] A. K. Gehi, R. Lampert, E. Veledar et al., "A twin study of metabolic syndrome and autonomic tone," Journal of Cardiovascular Electrophysiology, vol. 20, no. 4, pp. 422-428, 2009.

[25] P. K. Stein, J. I. Barzilay, P. P. Domitrovich et al., "The relationship of heart rate and heart rate variability to non-diabetic fasting glucose levels and the metabolic syndrome: the cardiovascular health study," Diabetic Medicine, vol. 24, no. 8, pp. 855-863, 2007.

[26] E. J. Brunner, H. Hemingway, B. R. Walker et al., “Adrenocortical, autonomic, and inflammatory causes of the metabolic syndrome: nested case-control study," Circulation, vol. 106, no. 21, pp. 2659-2665, 2002.

[27] C. J. Chang, Y. C. Yang, F. H. Lu et al., "Altered cardiac autonomic function may precede insulin resistance in metabolic syndrome," The American Journal of Medicine, vol. 123, no. 5, pp. 432-438, 2010.

[28] S. M. Pikkujämsä, H. V. Huikuri, K. E. Airaksinen et al., "Heart rate variability and baroreflex sensitivity in hypertensive subjects with and without metabolic features of insulin resistance syndrome," American Journal of Hypertension, vol. 11, no. 5, pp. 523-531, 1998. 
[29] A. S. Balcioğlu, S. Akinci, D. Çiçek et al., "Which is responsible for cardiac autonomic dysfunction in non-diabetic patients with metabolic syndrome: prediabetes or the syndrome itself?," Diabetes \& Metabolic Syndrome: Clinical Research \& Reviews, vol. 10, no. 1, pp. S13-S20, 2016.

[30] S. Guzzetti, E. Piccaluga, R. Casati et al., "Sympathetic predominance in essential hypertension: a study employing spectral analysis of heart rate variability," Journal of Hypertension, vol. 6, no. 9, pp. 711-717, 1988 .

[31] A. S. Menzes Jr., H. G. Moreira, and M. T. Daher, "Analysis of heart rate variability in hypertensive patients before and after treatment with angiotensin II-converting enzyme inhibitors," Arquivos Brasileiros de Cardiologia, vol. 83, no. 2, pp. 169172, 2004.

[32] R. Vesalainen, I. Kantola, K. Airaksinen, K. Tahvanainen, and T. Kaila, "Vagal cardiac activity in essential hypertension: the effects of metoprolol and ramipril," American Journal of Hypertension, vol. 11, no. 6, Part 1, pp. 649-658, 1998.

[33] M. Petretta, D. Bonaduce, F. Marciano et al., "Effect of 1 year of lisinopril treatment on cardiac autonomic control in hypertensive patients with left ventricular hypertrophy," Hypertension, vol. 27 , no. 3, pp. 330-338, 1996.

[34] C. M. Chern, H. Y. Hsu, H. H. Hu, Y. Y. Chen, L. C. Hsu, and A. C. Chao, "Effects of atenolol and losartan on baroreflex sensitivity and heart rate variability in uncomplicated Essential hypertension," Journal of Cardiovascular Pharmacology, vol. 47, no. 2, pp. 169-174, 2006.

[35] T. Kishi, Y. Hirooka, S. Konno, and K. Sunagawa, “Angiotensin II receptor blockers improve endothelial dysfunction associated with sympathetic hyperactivity in metabolic syndrome," Journal of Hypertension, vol. 30, no. 8, pp. 1646-1655, 2012.

[36] P. Pavithran, E. S. Prakash, T. K. Dutta, and T. Madanmohan, "Effect of antihypertensive drug therapy on short-term heart rate variability in newly diagnosed essential hypertension," Clinical and Experimental Pharmacology \& Physiology, vol. 37, no. 2, pp. e107-e113, 2010.

[37] T. Hamada, M. Watanabe, T. Kaneda et al., "Evaluation of changes in sympathetic nerve activity and heart rate in essential hypertensive patients induced by amlodipine and nifedipine," Journal of Hypertension, vol. 16, no. 1, pp. 111-118, 1998.

[38] J. de Champlain, M. Karas, P. Nguyen et al., "Different effects of nifedipine and amlodipine on circulating catecholamine levels in essential hypertensive patients," Journal of Hypertension, vol. 16, no. 9, pp. 1357-1369, 1998.

[39] R. Žaliūnas, J. Braždžionytè, V. Zabiela, and R. Jurkevičius, "Effects of amlodipine and lacidipine on heart rate variability in hypertensive patients with stable angina pectoris and isolated left ventricular diastolic dysfunction," International Journal of Cardiology, vol. 101, no. 3, pp. 347-353, 2005.

[40] B. M. Psaty, S. R. Heckbert, T. D. Koepsell et al., "The risk of myocardial infarction associated with antihypertensive drug therapies," JAMA, vol. 274, no. 8, pp. 620-625, 1995.

[41] M. Karas, Y. Lacourcière, A. R. LeBlanc et al., "Effect of the renin-angiotensin system or calcium channel blockade on the circadian variation of heart rate variability, blood pressure and circulating catecholamines in hypertensive patients," Journal of Hypertension, vol. 23, no. 6, pp. 1251-1260, 2005.
[42] D. S. Goldstein, O. Bentho, M. Y. Park, and Y. Sharabi, "Lowfrequency power of heart rate variability is not a measure of cardiac sympathetic tone but may be a measure of modulation of cardiac autonomic outflows by baroreflexes," Experimental Physiology, vol. 96, no. 12, pp. 1255-1261, 2011.

[43] S. M. Williams, A. Eleftheriadou, U. Alam, D. J. Cuthbertson, and J. P. H. Wilding, "Cardiac autonomic neuropathy in obesity, the metabolic syndrome and prediabetes: a narrative review," Diabetes Therapy, vol. 10, no. 6, pp. 1995-2021, 2019. 